\title{
Mobile Robot Self Localization based on Multi-Antenna-RFID Reader and IC Tag Textile
}

\author{
Yasutake Takahashi ${ }^{1}$, Yuta $\mathrm{Ii}^{2}$, Mi Jian ${ }^{3}$, Wang $\mathrm{Jun}^{3}$, Yoichiro Maeda ${ }^{1}$, Masahiko Takeda ${ }^{4}$, Ryuji Nakamura ${ }^{5}$, \\ Hiroaki Miyoshi ${ }^{5}$, Hidenori Takeuchi ${ }^{6}$, Yoshinori Yamashita ${ }^{7}$, Hiroshi Sano ${ }^{8}$ and Atsuji Masuda ${ }^{8}$
}

\begin{abstract}
This paper presents a self-localization system using multiple RFID reader antennas and High-Frequency RFID-tag textile floor for an indoor autonomous mobile robot. Conventional self-localization systems often use vision sensors and/or laser range finders and an environment model. It is difficult to estimate the exact global location if the environment has number of places that have similar shape boundaries or small number of landmarks to localize. It tends to take a long time to recover the self-localization estimation if it goes wrong at once. Vision sensors work hard in dark lighting condition. Laser range finder often fails to detect distance to a transparent wall. In addition, the self-localization becomes unstable if obstacles occlude landmarks that are important to estimate position of the robot. Door opening and closing condition affects the selflocalization performance.

Self-localization system based on reading RFID-tags on floor is robust against lighting condition, obstacles, furniture and doors conditions in the environment. Even if the arrangement of the obstacles or furniture in the environment is changed, it is not necessary to update the map for the self-localization. It can localize itself immediately and is free from well-known kidnapped robot problem because the RFID-tags give global position information. Conventional self-localization systems based on reading RFID-tags on floor often use only one RFID reader antenna and have difficulty of orientation estimation. We have developed a self-localization system using multiple RFID reader antennas and High-Frequency RFID-tag textile floor for an indoor autonomous mobile robot. Experimental results show the validity of the proposed methods.
\end{abstract}

\section{INTRODUCTION}

Recently, RFID (Radio Frequency IDentification) system have been studied for autonomous mobile robot selflocalization. RFID is a wireless non-contact system that uses radio-frequency electromagnetic fields to transfer data from

\footnotetext{
${ }^{1}$ Y. Takahashi and Y. Maeda are with Graduate School of Engineering, University of Fukui, 3-9-1, Bunkyo, Fukui, Fukui, 910-8507, Japan \{yasutake, maeda\}@ir.his.u-fukui.ac.jp

${ }^{2}$ Y. Ii is with Faculty of Engineering, University of Fukui, 3-9-1, Bunkyo, Fukui, Fukui, 910-8507, Japan yii@ir.his.u-fukui.ac.jp

${ }^{3} \mathrm{~J}$. Mi and J. Wang are with Faculty of Engineering, University of Beijing Information Science \& Technology, Haidian, Beijing, 100192, China \{jmi, jwang\}@ir.his.u-fukui.ac.jp

4 M. Takeda is with I.F.Sangyo Co.,Ltd., 4-11-22 Ninomiya, Fukui, Fukui, 910-0015, Japan m.takeda@if-s.co.jp

5 R. Nakamura and H. Miyoshi are with Art Finex Co.,Ltd., 6-1-33 Kamitabatacho, Sabae, Fukui, 916-0037, Japan, \{rnaka,miyoshi\}@art-tec.co.jp

6 H. Takeuchi is with UTIC Co., Ltd., 2-29-1 Haruechotabata, Sakai, Fukui, 919-0477, Japan, takeuchi@up-ut.com

7 Y. Yamashita is with University of Fukui Hospital, 23-3 Matsuokashimoaizuki, Eiheiji, Fukui, 910-1193, Japan, yyama@u-fukui.ac.jp

8 H. Sano and A. Masuda are with Industrial Technology Center of Fukui Prefecture, 61 Kawaiwashizukacho, Fukui, Fukui, 910-0102, Japan, \{sano, a_masuda\}@fklab.fukui.fukui.jp
}

a tag for automatic identification and/or tracking. RFID systems are commonly used for inventory control and inspection in production lines[1]. As a RFID system uses radio waves, unlike visual sensors, it is robust against change of lighting condition or obstacles. RFID based self-localization methods have been investigated so far as mentioned below.

There are two types of communication in RFID system. One uses radio waves and the other uses electromagnetic induction for communication between reader/writer and IC tags. A RFID system based on radio wave communication using UHF or SHF band realizes long distance communication[2]. It can also estimate distance between the antenna and the IC tag based on the intensity of the signal received by an antenna. Unfortunately, the intensity of the radio waves is affected by obstacles between the antennas and that IC tags, therefore, the distance estimation is not robust against the environmental changes. Yamamoto et al. [1] have proposed the RFID system for mobile robot self-localization. Unfortunately, the system can estimates the position of the robot too roughly to navigate the mobile robot in the room. Hähnel et al.[3] proposed to use a simplified probabilistic sensor model and Monte-Carlo localization with the RFID system and showed good localization performance during navigation. However, in general, distance estimation with a radio wave communication based RFID system often suffers from obstacles between the IC tag and the antennas so that the localization cannot be performed safely in an environment with many obstacles, especially, humans.

On the other hand, RFID systems based on HF[4][5] or LF[6] band use electromagnetic induction. The communication distance is short and less than several hundred [mm]. It is hard to estimate the distance between the antenna and the tags but it is quite accurate to detect the tag if the tag is within the range of the antenna. It rarely suffers from obstacles between the the IC tag and the antennas. Therefore, the position estimation is more accurate than the ones based on radio wave communication with UHF or higher frequency bands. Therefore, we choose HF band RFID system for our mobile robot self-localization system.

Conventional self-localization methods based on HF-band RFID systems[7][8] use a single antenna on bottoms of mobile robots to detect the tags on the floor. A RFID reader with one single antenna can detect tags in the region of the antenna but cannot estimate the position of the tags in the antenna/robot coordinate system. They can estimate the robot position based on the read tags but cannot estimate the orientation of the antenna/robot with the read tags in one 
sampling time. The need to read RFID tags data sequentially during the robot movement and a motion model of the vehicle to estimate the robot's orientation. Good initial estimated position and sufficient data are essential for accurate estimation. Another way to estimate the robot orientation with one antenna is to rotate the antenna with high speed on bottom of the robot and read the tags on the floor[9]. The system needs mechanical rotation mechanism and accurate tag reading system with high speed.

We propose a new self-localization system using multiple RFID reader antennas and High-Frequency RFID-tag textile. The RFID reader with multiple antennas enables to detect IC-tags on the floors and estimate not only the position of the robot but also orientation in one sampling time simultaneously. This paper shows two simple self-localization methods without probabilistic sensor or motion models as it shows the principal potentials of the multiple RFID reader antenna based self-localization system. The contributions of our system are

- establishment of loom technology of RFID-tags textile[10],

- development of HF band RFID reader with multiple antennas, and

- development of simple self-localization methods without any probabilistic motion models so that it can use not only active mobile robots but also passive carts.

This paper mainly focuses on the latter two points. Experimental results show the validity of the proposed system.

\section{RFID SYSTEM FOR SELF-LOCALIZATION}

Fig.1(a) shows a HF band RFID IC tag we adopt for our experiments. The size of the IC tag is $10 \times 20$ [mm]. Fig.1(b) shows a IC tag textile. The IC tags inserted automatically in one polyester sheet while weaving[10]. The interval of IC tags is about $10[\mathrm{~cm}]$. As the tags are inserted into the textile, there are some errors that can't be avoided. The positions of the tags are measured manually one by one, and then use those data build a IC tag map.

Fig.2(a) shows a carpet which is installed the IC tag textile sheet shown by Fig.1(b). It contains the polyester sheet shown in Fig.1(b). IC tags are not visible because the IC tag textile sheet is covered. We have installed the carpets into a room and a corridor adjacent to the room at a building of University of Fukui as shown in Fig.2(b) and Fig.2(c)

The sheet with the IC tags is installed on the floor and a mobile robot with a RFID reader runs on it (Fig.3(a)). The robot has a set of 8 RFID antennas on the bottom of the vehicle. Fig.3(b) shows an illustration of the set of 8 RFID antennas on bottom of the vehicle and IC tags on the floor. The RFID reader with 8 antennas reads the IC tags on the floor and find the IC tags' position information from the IC tag map. One antenna covers an area on the floor to detects the IC tags. The area is slightly larger than the antenna size.

We use a RFID reader with multiple antennas, ASI4300M3C(8CH) from ART FINEX and 8 RFID antennas. One antenna size is $60 \times 60[\mathrm{~mm}]$. We have attached 8 antennas to top of an acrylic plate and aluminum thin boards

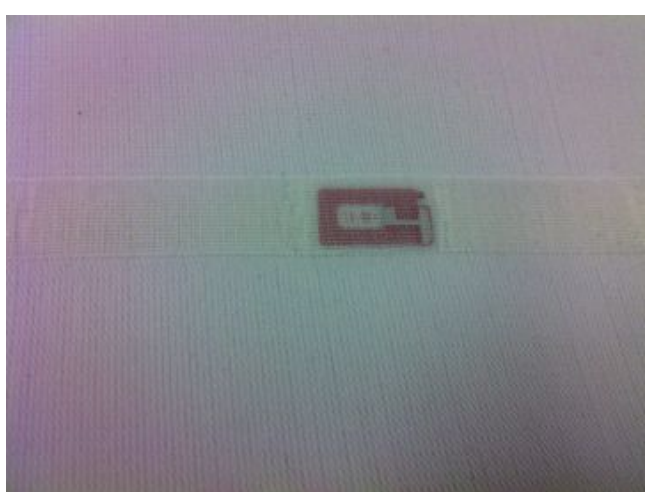

(a) IC Tag

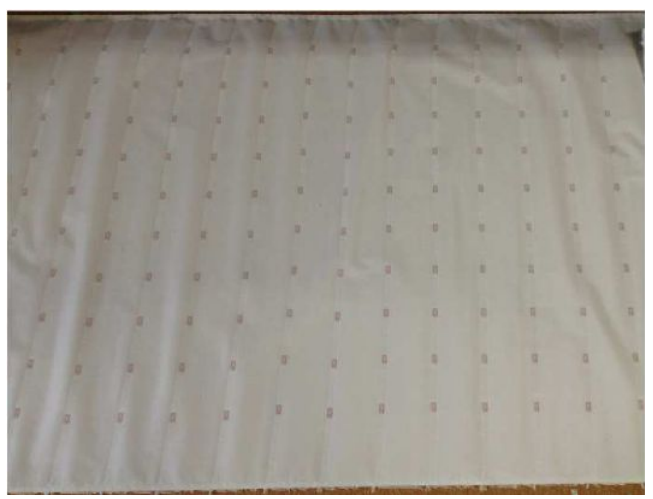

(b) IC Tag Textile

Fig. 1. IC tag textile

to the bottom of the plate as shown in Fig. 4(a) and (b). The distance between the antennas is $80[\mathrm{~mm}]$. The aluminum thin boards with 20 [mm] height are for limitation of IC tag detection region for each antenna. They prevent one antenna from reading a tag that is not closest to the antenna and lead the antenna to read the closest tag. The distance between the antennas and the floor ( $h$ in Fig.3(b)) is about 45 [mm] for the experiments.

\section{SElf-Localization BASED ON RFID SySTEM}

We propose two methods to estimate the robot position and orientation based on the RFID system described in previous section. We developed the methods as simple as possible because we are interested in basic self-localization performance of the multiple antennas RFID system without any probabilistic models. One method regards multiple small antennas as one large antenna to estimate position and the other uses antenna arrangement information for the position estimation. The former method needs less computational cost and the latter one gives more accurate location and posture estimation. We assume that the RFID IC tag map is known. The map contains tag location data providing position information ${ }^{w} \boldsymbol{p}_{t_{j}}$ of a IC tag $t_{j}$ in world coordinate system. 


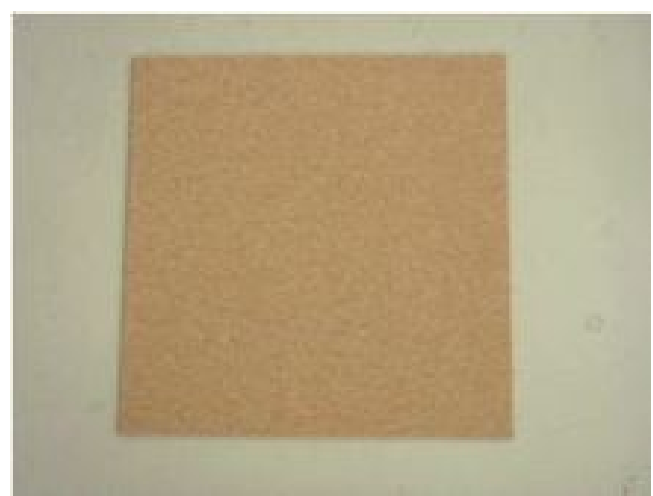

(a) IC Tag Textile Carpet

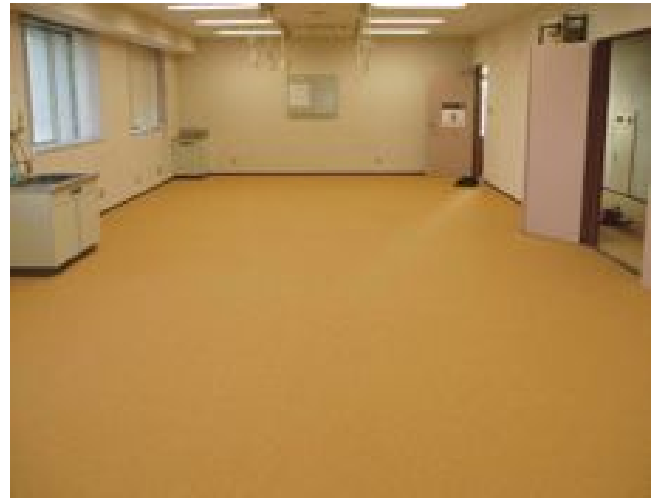

(b) Room installed IC Tag Textile Carpets

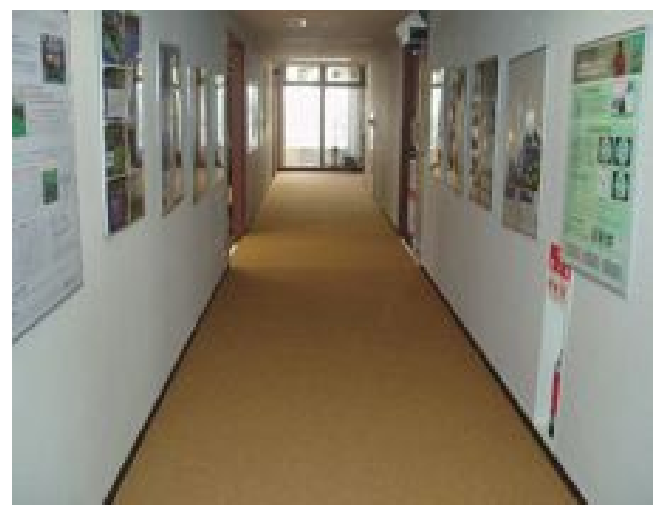

(c) Corridor installed IC Tag Textile Carpets

Fig. 2. IC Tag Textile Carpet and Installed Room and Corridor

\section{A. Method 1: Estimation without consideration on multiple antennas arrangement}

Method 1 estimates center position of the set of the antennas without consideration on the antennas arrangement. The center position and the orientation of the set of the antennas relative to the world coordinate system are ${ }^{w} \boldsymbol{p}_{A}=\left({ }^{w} x_{A},{ }^{w} y_{A}\right){ }^{T}$ and ${ }^{w} \theta_{A}$, relatively, as shown in Fig.5. ${ }^{w} \boldsymbol{p}_{t_{j}}=\left({ }^{w} x_{t_{j}},{ }^{w} y_{t_{j}}\right)^{T}$ is the position of the IC tag in world coordinate system. ${ }^{w} \boldsymbol{p}_{a_{i}}=\left({ }^{w} x_{a_{i}},{ }^{w} y_{a_{i}}\right)^{T}$ is the position of each antenna in world coordinate system. $n_{A}$ is the number of antennas. $D_{i}$ is the set of IC tags $t_{j}$ detected by the $i$ th antenna. Method 1 estimates the center position of the set of antennas as the average positions of the IC tags detected

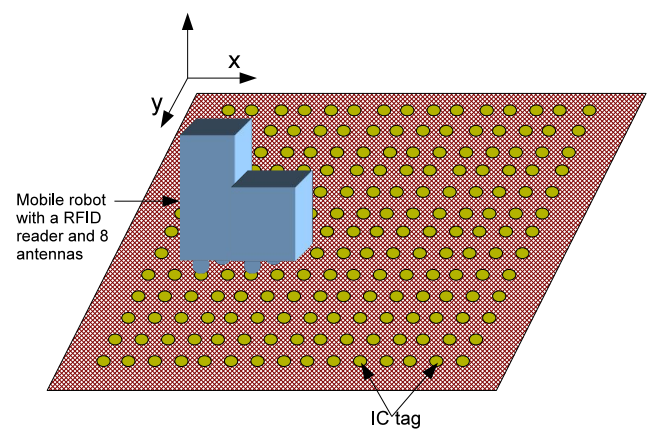

(a) A mobile robot with a RFID reader and IC Tag textile on floor

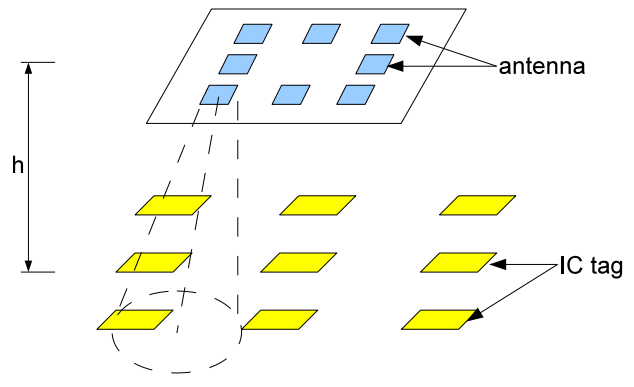

(b) 8 antennas and IC tags on the floor

Fig. 3. Localization System based on RFID

by the antennas by Eq. (1). In Fig.5, the blue point indicates the true position and the red point is the estimated position.

$$
\left({ }^{w} x_{A},{ }^{w} y_{A}\right)^{T}=\frac{\sum_{i=1}^{n_{A}} \sum_{j \in D_{i}}\left({ }^{w} x_{t_{j}},{ }^{w} y_{t_{j}}\right)^{T}}{n_{A}\left|D_{i}\right|}
$$

where $\left|D_{i}\right|$ indicates number of the IC tags detected by the $i$ th antenna. Method 1 takes the position of each antenna into consideration for the orientation estimation. The position of each antenna in antenna coordinate system ${ }^{A} \Sigma$ is ${ }^{A} \boldsymbol{p}_{a_{i}}=\left({ }^{A} x_{a_{i}},{ }^{A} y_{a_{i}}\right)^{T}$, and the angle of the antenna in ${ }^{A} \Sigma$ is ${ }^{A} \theta_{i}$. The orientation of ${ }^{A} \Sigma$ against ${ }^{w} \Sigma$ is calculated with Eqs.(2) and (3). Eq.(2) estimates the vector of the $\mathrm{x}$-axis of antenna coordinate system in world coordinate system $\left({ }^{w} x_{\theta_{A}}\right.$ ,$\left.{ }^{w} \theta_{A}\right)^{T}$, then, Eq.(3) estimates the orientation of the $\mathrm{x}$-axis ${ }^{w} \theta_{A}$.

$$
\begin{gathered}
\left(\begin{array}{l}
{ }^{w} x_{\theta_{A}} \\
{ }^{w} y_{\theta_{A}}
\end{array}\right)=\sum_{i=1}^{n_{A}} \sum_{j \in D_{i}}\left(\begin{array}{cc}
\cos ^{A} \theta_{i} & \sin ^{A} \theta_{i} \\
-\sin ^{A} \theta_{i} & \cos ^{A} \theta_{i}
\end{array}\right)\left(\begin{array}{l}
{ }^{w} x_{t_{j}}-{ }^{w} x_{A} \\
{ }^{w} y_{t_{j}}-{ }^{w} y_{A}
\end{array}\right) \\
{ }^{w} \theta_{A}=\arctan \left(\frac{{ }^{w} x_{\theta_{a}}}{{ }^{w} y_{\theta_{a}}}\right)
\end{gathered}
$$

\section{B. Method 2: Estimation with consideration on multiple antennas arrangement}

Method 2 takes multiple antennas arrangement into consideration for the self-localization. It is supposed to realize more accurate self-localization. The basic idea is to estimate the parameters of position and orientation of the antenna coordinate system in world coordinate system by minimizing 


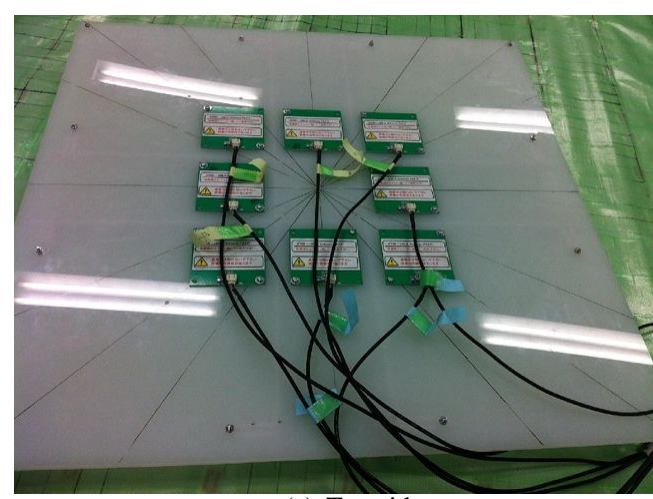

(a) Top side

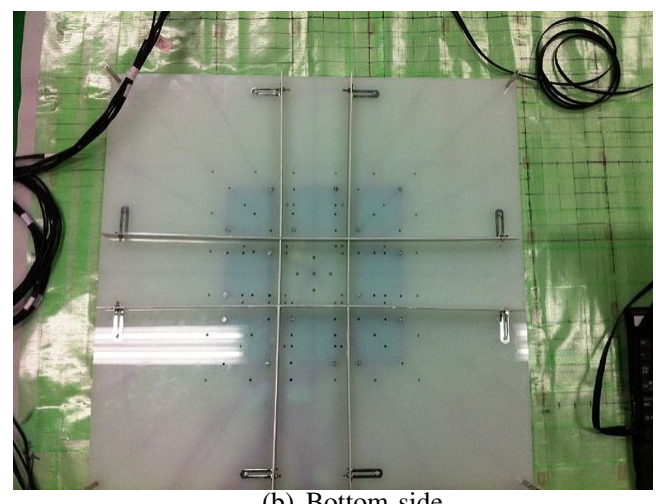

(b) Bottom side

Fig. 4. Antennas on an acrylic plate with partitions of aluminum boards

sum of distance errors between the detected IC tag and the antenna that detects the tag, as shown in Fig. 7.

The position of antenna $a_{i}$ is estimated by Eq.(4).

$$
\left(\begin{array}{c}
{ }^{w} x_{a_{i}} \\
{ }^{w} y_{a_{i}}
\end{array}\right)=\left(\begin{array}{cc}
\cos ^{w} \theta_{A} & \sin ^{w} \theta_{A} \\
-\sin ^{w} \theta_{A} & \cos ^{w} \theta_{A}
\end{array}\right)\left(\begin{array}{c}
{ }^{A} x_{a_{i}} \\
{ }^{A} y_{a_{i}}
\end{array}\right)+\left(\begin{array}{c}
{ }^{w} x_{A} \\
{ }^{w} y_{A}
\end{array}\right)
$$

The square distance error $E$ between the detected IC tag and the antenna that detects the tag in world coordinate system is calculated by Eq.(5).

$$
E=\sum_{i=1}^{n_{A}} \sum_{j \in D_{i}}\left|{ }^{w} \boldsymbol{p}_{t_{j}}-{ }^{w} \boldsymbol{p}_{a_{i}}\right|^{2}
$$

where ${ }^{w} \boldsymbol{p}_{a_{i}}=\left({ }^{w} x_{a_{i}},{ }^{w} y_{a_{i}}\right)^{T}$ and ${ }^{w} \boldsymbol{p}_{t_{j}}$ is position of detected IC tag in world coordinate system.

A simplified Gibbs Sampling method gives a proper way to find ${ }^{w} \boldsymbol{p}_{A}$ and ${ }^{w} \theta_{A}$ that minimizes the square distance error $E$. The algorithm is shown below:

Step $1\left({ }^{w} x_{A},{ }^{w} y_{A},{ }^{w} \theta_{A}\right)$ is initialized by Method 1 .

Step 2 Find $\Delta x$ that minimizes $E$ in Eq.(5) with $\left({ }^{w} x_{A}+\right.$ $\left.\Delta x,{ }^{w} y_{A},{ }^{w} \theta_{A}\right)$ by sampling $E$ changing $\Delta x$ from minimum to maximum by predefined sampling value.

Step 3 Replace the estimated position of $\mathrm{x}$-coordinate ${ }^{w} x_{A} \leftarrow{ }^{w} x_{A}+\Delta x$.

Step 4 Find $\Delta y$ that minimizes $E$ in Eq.(5) with $\left({ }^{w} x_{A},{ }^{w} y_{A}+\Delta y,{ }^{w} \theta_{A}\right)$ by sampling $E$ changing $\Delta x$ from minimum to maximum by predefined sampling value.

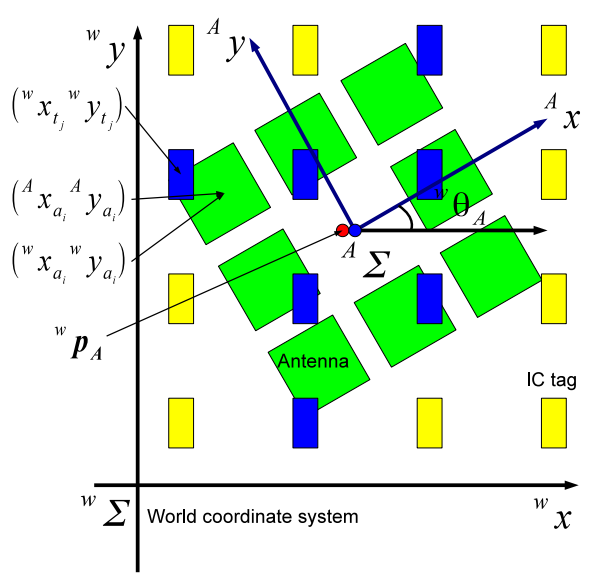

Fig. 5. Illustration of world coordinate system ${ }^{w} \Sigma$ and antenna coordinate system ${ }^{A} \Sigma$

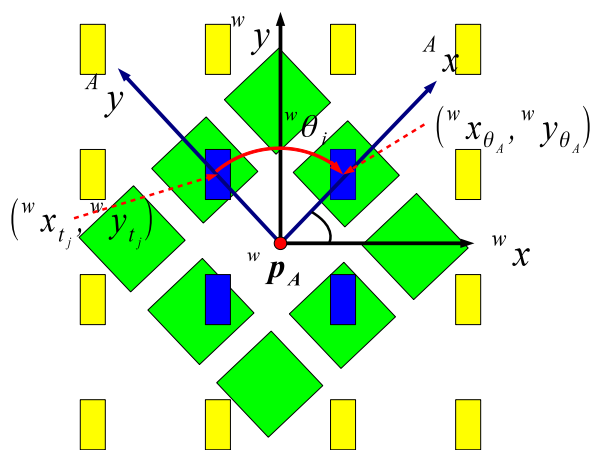

Fig. 6. Orientation estimation in Method 1

Step 5 Replace the estimated position of $\mathrm{y}$-coordinate ${ }^{w} y_{A} \leftarrow{ }^{w} y_{A}+\Delta y$.

Step 6 Find $\Delta \theta$ that minimizes $E$ in Eq.(5) with $\left({ }^{w} x_{A},{ }^{w} y_{A},{ }^{w} \theta_{A}+\Delta \theta\right)$ by sampling $E$ changing $\Delta x$ from minimum to maximum by predefined sampling value.

Step 7 Replace the estimated orientation ${ }^{w} \theta_{A} \leftarrow{ }^{w} \theta_{A}+\Delta \theta$.

Step 8 If $(\Delta x, \Delta y, \Delta \theta) \neq(0,0,0)$, goto step 2

The algorithm above finds adequate solution with much smaller cost than a full search method.

\section{SElF-LocalizATION ExPERIMENTS}

\section{A. Evaluation experiment procedures}

A set of 8 antennas on an acrylic plate with aluminum thin boards shown in Fig. 4(a) and (b) is put on the IC tag textile with 45 [mm] height. Self-localization based on Methods 1 and 2 is performed while moving parallel to the $x$ and $y$ axes in the world coordinate system. We sample data every 20 $[\mathrm{mm}]$ from $(\mathrm{x}, \mathrm{y})=(300,300)[\mathrm{mm}]$ to $(\mathrm{x}, \mathrm{y})=(600,600)[\mathrm{mm}]$. Self-localization is performed 10 times at each sampling point to evaluate the error of position and orientation in world coordinate system. 


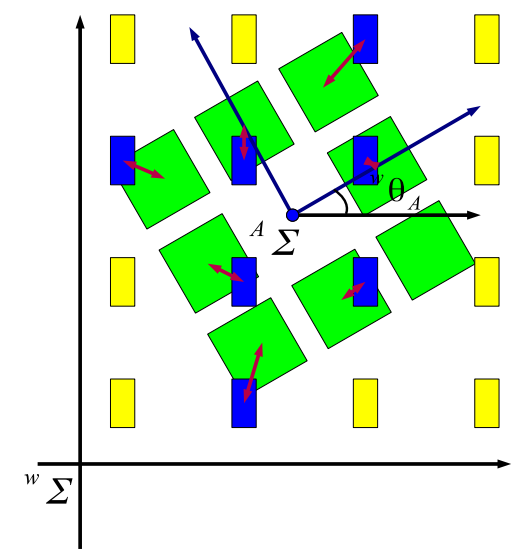

Fig. 7. Illustration of position and orientation estimation of Method2

\section{B. Experiment results}

Figs. 8, 9 and 10 show the self-localization error by Methods 1 and 2 when the antenna's orientation is $0^{\circ}, 30^{\circ}$ and $45^{\circ}$ to world coordinate system. Table I shows the average and maximum estimation errors in all cases. The table shows that the aluminum partition successfully reduces the estimation error. The figures and the table show that Method 1 has less than $26[\mathrm{~mm}]$ error for parallel displacement and less than 0.113 [rad] error for orientation on average. Maximum error becomes big at number of points. The reason seems to be that the RFID reader detects only few IC tags at the points while it detects more IC tags at the other points. Method 2 shows better estimation performance than the Method 1. It suggests that multiple antennas and the antenna-arrangement information offers better estimation of localization.

\section{Real-time self-localization experiment}

We carry out real-time self-localization experiment using the RFID reader with multiple antennas in order to evaluate the system for a mobile robot. We move the antennas on the acrylic board parallel to the $\mathrm{x}$ and $\mathrm{y}$ axes of world coordinate system. It starts from $(x, y)=(300,300)[\mathrm{mm}]$ and go to $(x, y)=(300,600)[\mathrm{mm}],(x, y)=(600,600)[\mathrm{mm}],(x, y)=$ $(600,300)[\mathrm{mm}]$, and start position, again. It goes on straight lines from start and end points. It repeats the route 5 times. The trajectories are logged during the movement as shown in Fig. 11. The blue line in the figure is the estimated position during the movement, and the black arrow indicates the orientation of antenna's plate. Self-localization with proposed methods works stably although the estimation error becomes bigger if the orientation of antenna's plate is close to $45^{\circ}$. We confirm from the results that it is possible to use this self-localization system for a mobile robot.

\section{COnClusions And Future Work}

In this paper, we proposed methods of self-localization based on RFID reader with multiple antennas and HF band IC tag textile and verified the performance of the proposed methods. HF band RFID system enables the self-localization

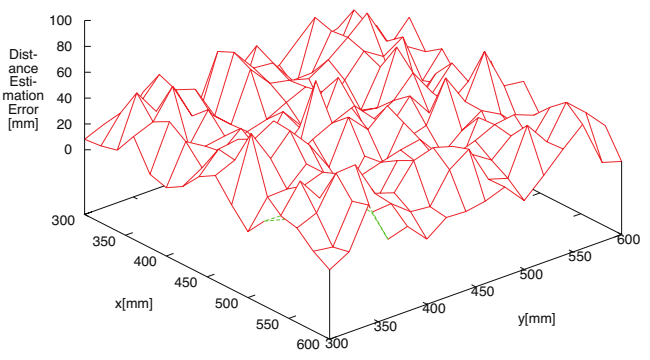

(a) Position estimation error by Method 1

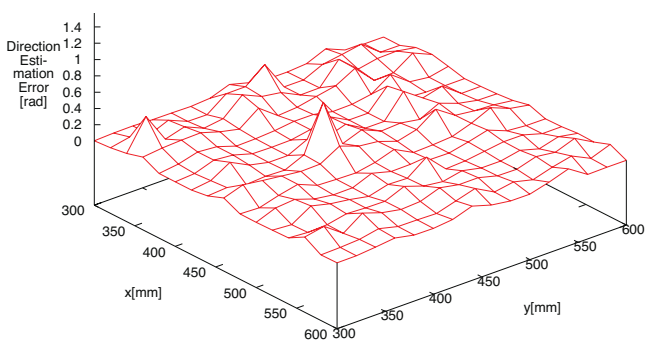

(b) Orientation Estimation error by Method 1

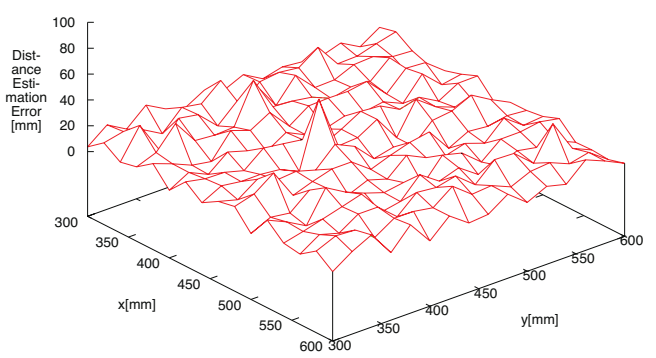

(c) Position estimation error by Method 2

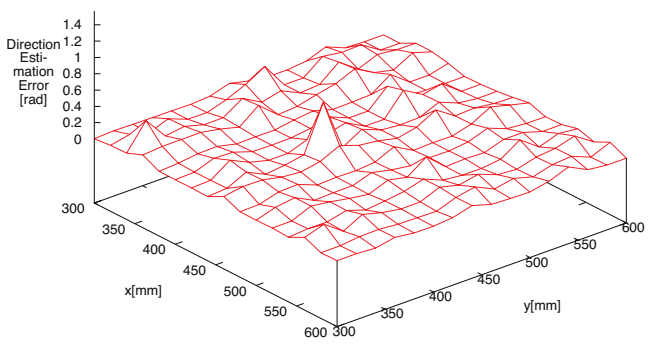

(d) Orientation estimation error by Method 2

Fig. 8. Self-localization error in case antenna's orientation is $0^{\circ}$

more robust against lighting condition, moving obstacles and furniture, and transparent walls. The RFID reader with multiple antennas enables to estimate not only position on the field but also orientation simultaneously without any other sensors. We confirmed that the self-localization becomes more accurate by using the position information of each antenna.

The proposed localization system is robust against lighting condition Vision sensors work hard in dark lighting condition. Laser range finder often fails to detect distance to a transparent wall. In addition, the self-localization becomes unstable if obstacles occlude landmarks that are important to estimate position of the robot. Door opening and closing condition affects the self-localization performance. 
TABLE I

ESTIMATION ERROR OF EACH METHOD OF SELF-LOCALIZATION

\begin{tabular}{|c|c|c|c|c|c|c|c|c|}
\hline Item & \multicolumn{2}{|c|}{ Without partition } & \multicolumn{6}{|c|}{ With partition of aluminum } \\
\hline Height from the ground to the antenna & \multicolumn{8}{|c|}{$45[\mathrm{~mm}]$} \\
\hline Posture angle of antenna & \multicolumn{2}{|c|}{$0^{\circ}$} & \multicolumn{2}{|c|}{$0^{\circ}$} & \multicolumn{2}{|c|}{$30^{\circ}$} & \multicolumn{2}{|c|}{$45^{\circ}$} \\
\hline Method & Method 1 & Method 2 & Method 1 & Method 2 & Method 1 & Method 2 & Method 1 & Method 2 \\
\hline Average position estimation error $[\mathrm{mm}]$ & 35.7 & 19.0 & 26.0 & 13.0 & 18.4 & 13.9 & 23.8 & 16.7 \\
\hline Maximum position estimation error $[\mathrm{mm}]$ & 82.7 & 69.3 & 66.2 & 53.3 & 69.6 & 51.0 & 72.1 & 48.3 \\
\hline Average orientation estimation error [rad] & 0.147 & 0.132 & 0.072 & 0.065 & 0.113 & 0.112 & 0.099 & 0.091 \\
\hline Maximum orientation estimation error [rad] & 1.112 & 1.051 & 0.677 & 0.651 & 0.419 & 0.369 & 0.310 & 0.288 \\
\hline
\end{tabular}

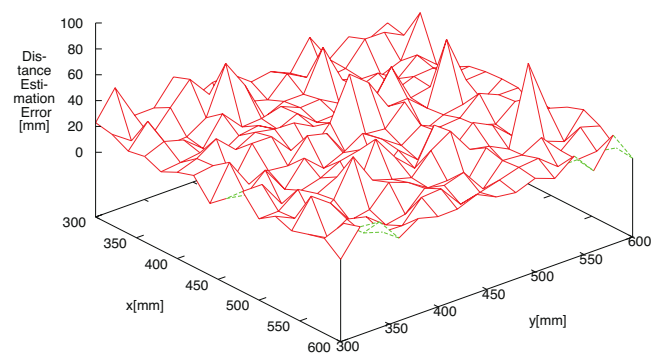

(a) Position estimation error by Method 1

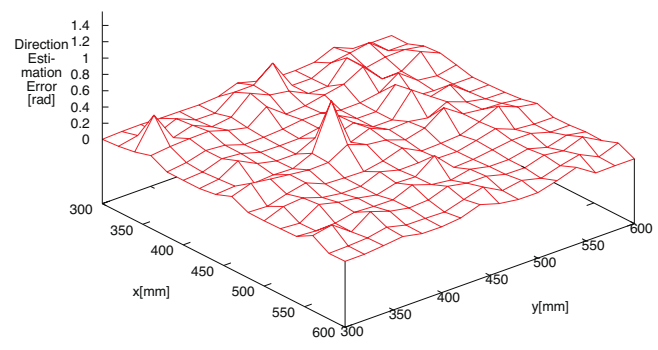

(b) Orientation estimation error by Method 1

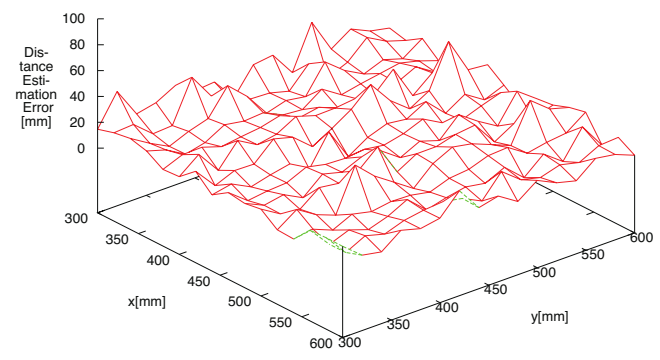

(c) Position estimation error by Method 2

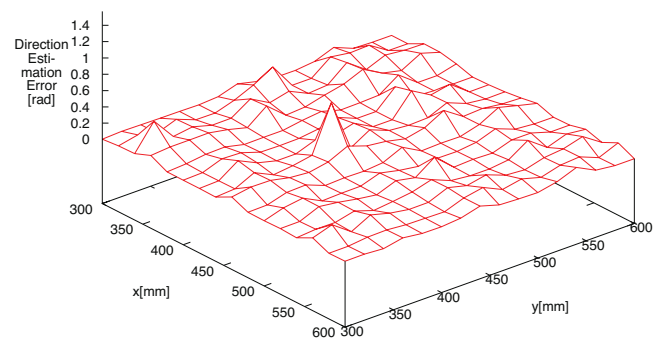

(d) Orientation estimation error by Method 2

Fig. 9. Self-localization error in case antenna's angle is $30^{\circ}$

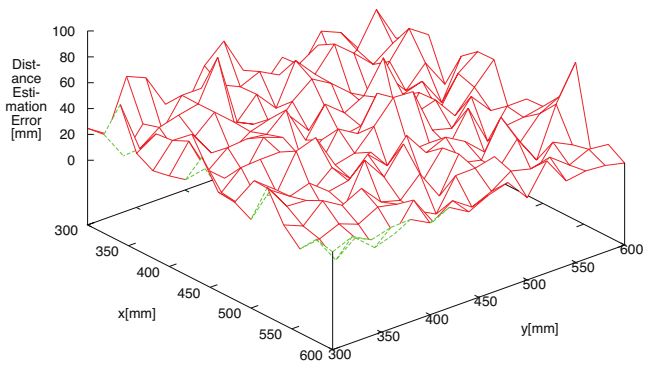

(a) Position estimation error by Method 1

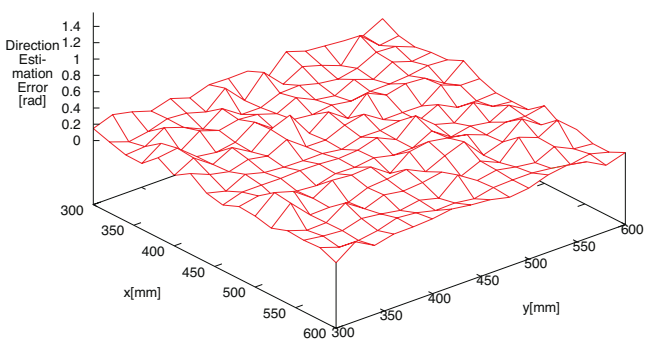

(b) Orientation estimation error by Method 1

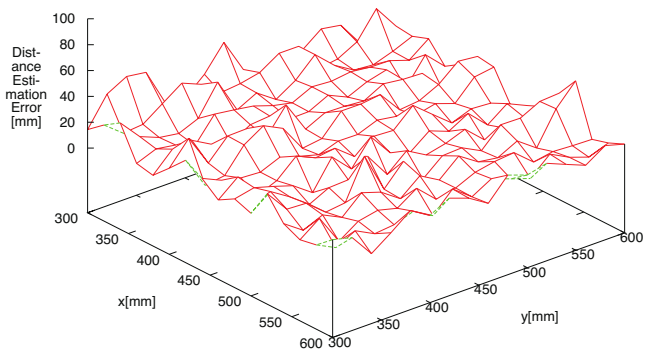

(c) Position estimation error by Method 2

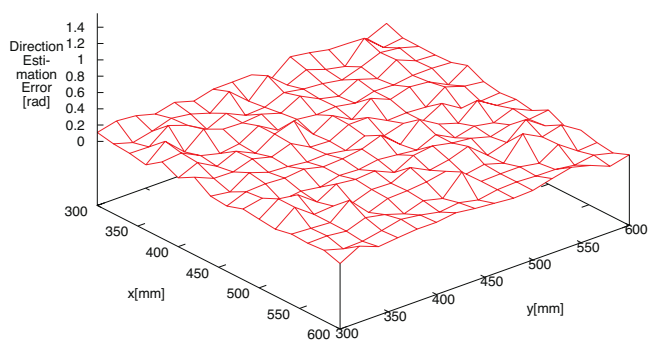

(d) Orientation estimation error by Method 2

Fig. 10. Self-position estimation error as antenna's angle is $45^{\circ}$ 


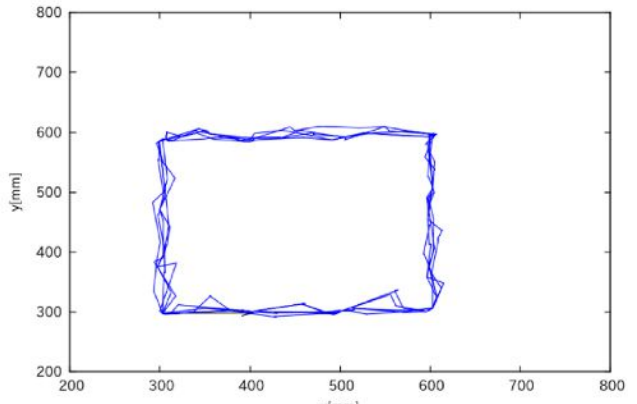

(a) Trajectory of self-localization in the state of the antenna orientation angle of $0^{\circ}$

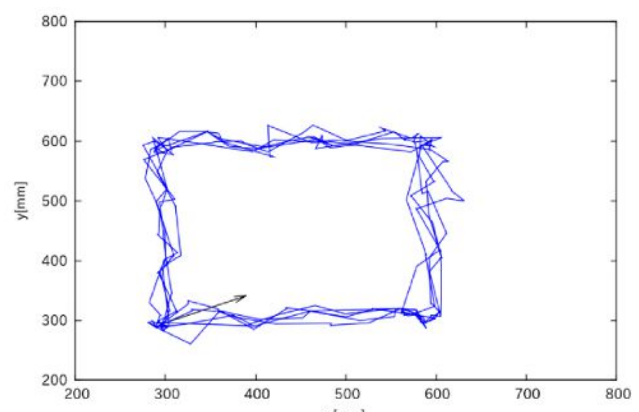

(b) Trajectory of self-localization in the state of the antenna orientation angle of $30^{\circ}$

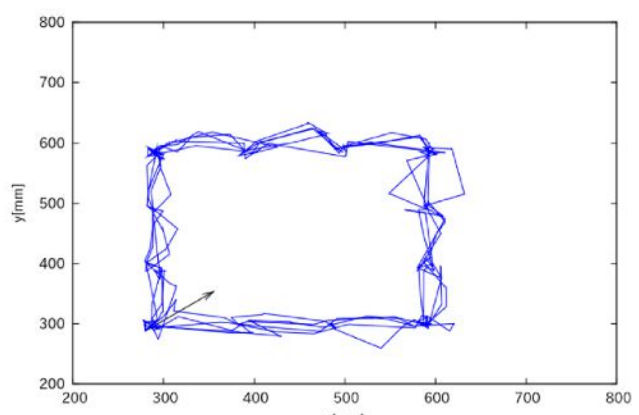

(c) Trajectory of self-localization in the state of the antenna orientation angle of $45^{\circ}$

Fig. 11. The trajectory of position estimation using the antenna height of $45[\mathrm{~mm}]$ from the surface of the IC tag

The proposed self-localization system based on reading RFID-tags on floor is robust against lighting condition, obstacles, furniture and doors conditions in the environment. It does not suffer from dark lighting condition or a transparent wall. The self-localization becomes stable even if obstacles occlude landmarks that are important to estimate position of the robot for conventional vision-based or laser-rangefinder-based system. Door opening and closing condition does not affect the self-localization performance of the proposed system. Even if the arrangement of the obstacles or furniture in the environment is changed, it is not necessary to update the map for the self-localization. It can localize itself immediately and is free from well-known kidnapped robot problem because the RFID-tags give global position information.
The installation cost of IC-tag textiles is mostly limited to the cost of the RFID-IC tag. Development and installation of the IC-tag textiles are not hard because the techniques for inserting the IC tags automatically have been developed [10] and the IC-tag textiles are commercially available.

The experiments show that the arrangement of RFID antennas affects the localization estimation. The arrangement design to detect more number of IC tags with small number of antennas is future work. It might reduce the maximum estimation error at some points in the experiment as the arrangement and interval of the antenna is important for localization. Another way to reduce the maximum estimation error might be to use extended Kalman filter or some other probabilistic models as Hähnel et al.[3] proposed although we were interested in the basic performance of the RFID system in this paper. It is necessary to develop more accurate self-localization method. Furthermore, automatic RFID Tag map building is future work, too.

\section{ACKNOWLEDGEMENT}

A part of this work was supported by Supporting Industries Operation of Ministry of Economy, Trade and Industry, Japan.

\section{REFERENCES}

[1] K. Yamano, K. Tanaka, M. Hirayama, E. Kondo, Y. Kimuro, and M. Matsumoto, "Self-localization of mobile robots with rfid system by using support vector machine," in Proceedings of 2004 IEEE/RSJ International Conference on Intelligent Robots and Systems, 2004, pp. $3756-3761$.

[2] S. Schneegans, P. Vorst, and A. Zell, "Using rfid snapshots for mobile robot self-localization," in European Conference on Mobile Robots, 2007, pp. 241-246.

[3] D. Hähnel, W. Burgard, D. Fox, K. P. Fishkin, and M. Philipose, "Mapping and localization with rfid technology," in Proceedings of the 2004 IEEE International Conference on Robotics \& Automation, 2004, pp. 1015-1020.

[4] U. Tomohiro, M. Yasushi, I. Kenji, A. Tatsuo, and Y. Jun-ichi, "Pose estimation of objects using multiple id devices," Journal of the Robotics Society of Japan, vol. 23, no. 1, pp. 84-94, 2005.

[5] S. Masahiro, K. Takayuki, E. Daniel, I. Hiroshi, and H. Norihiro, "Communication robot for science museum with rfid tags," Journal of the Robotics Society of Japan, vol. 24, no. 4, pp. 489-496, 2006.

[6] M. Kouji, H. Tsutomu, K. Yoshihiko, S. Yosuke, I. Takafumi, A. Daisaku, and K. Ryo, "A method to manage data flow between intelligent robots and an intelligent environment," Journal of the Robotics Society of Japan, vol. 26, no. 2, pp. 192-199, 2008.

[7] S. Park and S. Hashimot, "Autonomous mobile robot navigation using passive rfid in indoor environment," IEEE TRANSACTIONS ON INDUSTRIAL ELECTRONICS, vol. 56, no. 7, pp. 2366-2373, 2009.

[8] S. Han, H. Lim, and J. Lee, "An efficient localization scheme for a differential-driving mobile robot based on rfid system," IEEE TRANSACTIONS ON INDUSTRIAL ELECTRONICS, vol. 54, no. 6, pp. 3362$3369,2007$.

[9] T. Asakawa, K. Nishihara, and T. Yoshidome, "A detection system of location and direction angle by a rf tag reader using a rotary antenna," Transactions of the Japan Society of Mechanical Engineers, vol. 20, no. 1, pp. 189-195, 2008.

[10] A. Masuda, T. Murakami, R. Ueoka, and M. Hirose, "The development of uhf ic tag textile," in Proceedings of the 40th Textile Research Symposium at Kyoto, 2011, pp. 99-102. 